\title{
PERIODIC POINTS OF LATITUDINAL SPHERE MAPS
}

\author{
MICHAE MISIUREWICZ
}

\begin{abstract}
For the maps of the two-dimensional sphere into itself that preserve the latitude foliation and are differentiable at the poles, lower estimates of the number of fixed points for the maps and their iterates are obtained. Those estimates also show that the growth rate of the number of fixed points of the iterates is larger than or equal to the logarithm of the absolute value of the degree of the map.
\end{abstract}

\section{INTRODUCTION}

Pugh and Shub [4] introduced an interesting class of maps of the two-dimensional sphere $\mathbb{S}^{2}$ to itself. These are maps of class $C^{1}$ that preserve the latitude foliation. They studied the growth rate of the number of fixed points of iterates of the map and compared it to the degree of the map. This problem is connected with the Entropy Conjecture [5], its special case involving the degree of the map [3], and the relation between entropy and horseshoes [1]. The main result of [4] is that if $f: \mathbb{S}^{2} \rightarrow \mathbb{S}^{2}$ is a map of class $C^{1}$ preserving the latitude foliation, then for each $n$ the map $f^{n}$ has at least $2^{n}$ fixed points.

The aim of this paper is to extend and explain the results of [4]. The reason why $f^{n}$ has at least $2^{n}$ fixed points is that the degree of $f^{n}$ is $2^{n}$ and every map of degree $2^{n}$ from the class of map we consider has at least $2^{n}+1$ fixed points. Moreover, the minimal possible growth rate the number of fixed points of the $n$-th iterate of such map can be expressed in the terms of the degree and direction of bands, the objects introduced in [4], and is often larger that the logarithm of the absolute value of the degree of the map. Additionally, the assumptions on the map can be weakened; differentiability is necessary only at the poles.

The maps considered here resemble triangular maps (see, e.g., [2]), that is, skew products with a closed interval both in the base and in the fiber. Here the fibers are circles instead of the intervals, and the fibers over the endpoints of the base interval are degenerated to points.

The paper is organized as follows. In Section 2 notation is introduced and basic facts are presented. In Section 3 lower estimates of the number of fixed points of the iterates of our maps and of the growth rate of this number are obtained. Those estimates are used to prove that if the absolute value of the degree is larger than 1 , then its logarithm is not larger than the mentioned growth rate. In Section 4 it is proven that for the maps under consideration the number of the fixed points is at least the absolute value of the degree of the map plus 1 . This gives another proof for

Date: March 2, 2014.

2010 Mathematics Subject Classification. Primary: 37E30, 37C25.

Key words and phrases. Sphere maps, periodic points. 
the inequality between the growth rate and the logarithm of the degree. In Section 5 some examples are given.

\section{Preliminaries}

The notation and terminology used here will be slightly different than the one used in [4]. Mainly it will be more geographical.

We take the spherical coordinates on the unit sphere $\mathbb{S}^{2}$ in $\mathbb{R}^{3}$. One of the components of these coordinates is latitude. For simplicity, we rescale it affinely, so that it varies from 0 (at the South Pole) to 1 (at the North Pole). We denote the latitude of a point $x \in \mathbb{S}^{2}$ by $\ell(x)$. This defines a function $\ell: \mathbb{S}^{2} \rightarrow[0,1]$. For every $a \in(0,1)$ the set $\ell^{-1}(a)$ is a circle; we call it a parallel. The set $\ell^{-1}(0)$ consists of the South Pole, while the set $\ell^{-1}(1)$ consists of the North Pole. Thus, we can speak of the latitude foliation, although, strictly speaking, it is not a foliation (because of the poles).

Definition 2.1. A map $f: \mathbb{S}^{2} \rightarrow \mathbb{S}^{2}$ will be called latitudinal if it preserves the latitude foliation (that is, if $\ell(x)=\ell(y)$ then $\ell(f(x))=\ell(f(y))$ ). We will denote the family of all latitudinal maps differentiable at the poles by $\mathcal{L}$.

Fix a latitudinal map $f$. Since it preserves the latitude foliation, there is a continuous map $\varphi:[0,1] \rightarrow[0,1]$ such that $\varphi \circ \ell=\ell \circ f$. For every interval $[a, b] \subset[0,1]$ such that $\varphi^{-1}(\{0,1\})=\{a, b\}$, we call the set $B=\ell^{-1}([a, b])$ a band (see Figure 1 ). It has three numbers assigned to it. The first one is the sign of $B$ and will be denoted $\Delta(B)$. It is +1 if $\varphi(a)=0$ and $\varphi(b)=1$; it is -1 if $\varphi(a)=1$ and $\varphi(b)=0$. The second one is the latitude degree of $B$, denoted $d(B)$, and it is defined as the degree of the circle map $\left.f\right|_{\ell^{-1}(c)}$ for any $c \in(a, b)$ (we give all parallels the same orientation). It does not depend on the choice of $c$ because all those circle maps in a band are homotopic to each other. Finally, we define the degree of $B$ as $\operatorname{deg}(B)=\Delta(B) \cdot d(B)$.

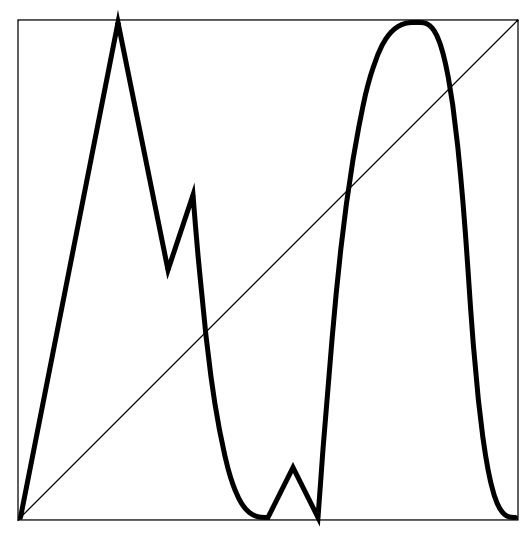

Figure 1. The map $\varphi$ for a latitudinal map with four bands.

We will denote the set of all bands of $f$ by $\mathcal{B}(f)$. It is clear that this set is finite. The following fact is proved in [4] (as Proposition 4).

Lemma 2.2. If $f: \mathbb{S}^{2} \rightarrow \mathbb{S}^{2}$ is a latitudinal map, then its degree is

$$
\operatorname{deg}(f)=\sum_{B \in \mathcal{B}(f)} \operatorname{deg}(B) .
$$


Denote by $N(f)$ the number of fixed points of $f$. We want to find the minimal possible number of fixed points of $f$, given the numbers $\Delta(B)$ and $d(B)$ for each band $B \in \mathcal{B}(f)$. Each fixed point of $f$ which is not a pole, is contained in an invariant parallel. Invariant parallels are preimages under $\ell$ of fixed points of $\varphi$ which are not 0 or 1 . We do not know anything about the invariant parallel outside the bands. In each band that does not contain a pole there must me at least one invariant parallel. The same is true for a band that contains a pole, but this pole is not a fixed point. However, if a pole is a fixed point, it may happen that the band containing it has no invariant parallel. In [4], this problem is addressed in Lemma 6. Here we need a slightly stronger statement, so we will reprove it. For easy reference, we include the trivial part.

Lemma 2.3. Let $f \in \mathcal{L}$ and let $B$ be a band of $f$. Then:

(a) if $B$ does not contain a pole which is a fixed point of $f$, then there is an invariant parallel in $B$,

(b) if $B$ contains a pole which is a fixed point of $f$, and $|d(B)| \neq 1$, then there is an invariant parallel in $B$.

Proof. Part (a) is obvious. Let us prove part (b). Let us take a smooth chart in a neighborhood of a pole which is in $B$, in which the pole goes to the origin in $\mathbb{R}^{2}$ and the parallels go to circles centered at the origin. Then the derivative at the pole becomes a linear map of $\mathbb{R}^{2}$ that preserves the foliation into those circles, or maps everything to 0 . In the first case, the degree of the derivative on those circles is \pm 1 , so the degree of the map $f$ represented in this chart on sufficiently small circles is also \pm 1 . This means that $|d(B)|=1$. Thus, if $|d(B)| \neq 1$ then the derivative is 0 , so $\varphi$ has derivative 0 at the corresponding endpoint of $[0,1]$. If $B=\mathbb{S}^{2}$, this applies to both poles. A continuous interval map from $[a, b] \subset[0,1]$ onto $[0,1]$ that has derivative 0 at every endpoint of $[a, b]$ which is a fixed point, has to have a fixed point in its interior (see Figure $2(\mathrm{~b})$ ). Therefore in this case there is an invariant parallel in $B$.

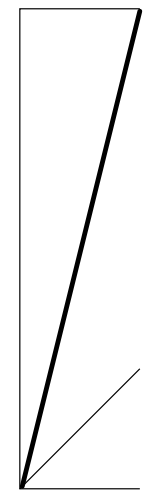

(a)

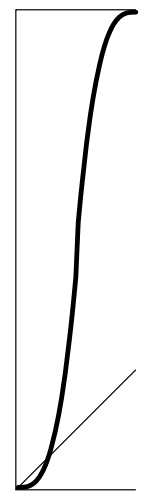

(b)

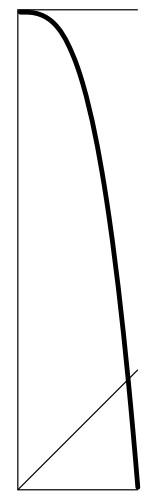

(c)

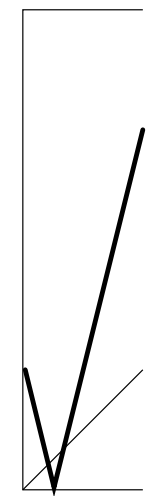

(d)

Figure 2. Some possibilities for $\varphi$ in a neighborhood of 0 .

We will use all the time the following well known fact. 
Lemma 2.4. A continuous map of the circle into itself of degree $d$ has at least $|d-1|$ fixed points.

\section{Fixed points of iterates}

Our aim in this section is to estimate the minimal possible number of fixed points of $f^{n}$, where $f$ is a latitudinal map, in terms of the latitude degrees of its bands. In particular, we are interested in the minimal possible growth rate of the number of fixed points of $f^{n}$, as $n \rightarrow \infty$. In this estimate the signs of the bands are irrelevant. Therefore we will introduce another kind of degree for $f$. Namely, the unsigned degree of $f$ will be

$$
u(f)=\sum_{B \in \mathcal{B}(f)}|d(B)|=\sum_{B \in \mathcal{B}(f)}|\operatorname{deg}(B)| .
$$

Let us note that it is easy (using symbolic dynamics or the counting preimages argument) to see that the topological entropy of $f$ is at least $\log u(f)$. If $k(f)$ is the number of bands of $f$, then the topological entropy of $f$ is also larger than or equal to the topological entropy of $\varphi$, which in turn is at least $\log k(f)$.

Now we are ready to prove the main theorem of this section.

Theorem 3.1. For a given $f \in \mathcal{L}$, let $u=u(f), k=k(f)$, and let $k_{+}$be the number of bands of $f$ of positive latitude degree, and $k_{-}$the number of bands of $f$ of negative latitude degree. Then

$$
N\left(f^{n}\right) \geq k^{n}-\left(k_{+}+k_{-}\right)^{n}+u^{n}-\left(k_{+}-k_{-}\right)^{n}+c_{n},
$$

where $c_{n}$ depends on the first and last bands of $f$, and $\left|c_{n}\right| \leq 2$. In particular,

$$
N\left(f^{n}\right) \geq k^{n}-\left(k_{+}+k_{-}\right)^{n}+u^{n}-\left(k_{+}-k_{-}\right)^{n}-2 .
$$

Proof. Let us first make computations ignoring problems caused by the poles. We will take them into account later, and they will produce the correction $c_{n}$.

By Lemma 2.4, each $f^{n}$-invariant parallel gives us at least $|d-1|$ fixed points of $f^{n}$, where $d$ is the degree of $f^{n}$ restricted to this parallel. If this invariant parallel is contained in a band of $f^{n}$, this degree is the latitude degree of the band.

We will treat $\mathcal{B}(f)$ as an alphabet. For a word $B_{0} B_{1} \ldots B_{n-1}$ there is a band $D \in \mathcal{B}\left(f^{n}\right)$ such that $f^{i}(D) \subset B_{i}$ for $i=0,1, \ldots, n-1$. We will say it is a band corresponding to this word. Its latitude degree for $f^{n}$ is equal to the product of latitude degrees for $f$ of $B_{i}, i=0,1, \ldots, n-1$. This means that this word contributes at least that many fixed points to $N\left(f^{n}\right)$ (remember that we ignore the problem with the poles). To simplify the terminology, we will speak of degrees of words.

Let us first count the fixed points of $f^{n}$ contributed by the words of degree 0 . Each such word contributes one fixed point, so the contribution is equal to the number of words of length $n$ of degree 0 . The simplest way to count them is to count all words and subtract the number of words of non-zero degree. In this way we get $k^{n}-\left(k_{+}+k_{-}\right)^{n}$.

Now count the fixed points of $f^{n}$ contributed by the words of non-zero degree. For each such word this number is the absolute value of the degree of the word, plus 1 if the degree is negative, or minus 1, if the degree is positive. Summing over all words of length $n$, we get the sum of absolute values of the degrees of all words of length $n$ (this is $u^{n}$, because the sum of all products is the product of the sums), plus the 
number of words of length $n$ of negative degree minus the number of words of length $n$ of positive degree. However, the sign of the degree is the product of the signs, so we can again switch from the sum of products to the product of sums (of plus or minus ones), and we see that we subtract $\left(k_{+}-k_{-}\right)^{n}$. This way we get $u^{n}-\left(k_{+}-k_{-}\right)^{n}$ fixed points.

Thus, we get $k^{n}-\left(k_{+}+k_{-}\right)^{n}+u^{n}-\left(k_{+}-k_{-}\right)^{n}$ as the total number of fixed points of $f^{n}$ contributed by all words of length $n$.

Let us now consider the poles. According to Lemma 2.3, the only cases when we may have to modify our estimates are when there is $D \in \mathcal{B}\left(f^{n}\right)$ that contains a pole that is fixed by $f^{n}$ and such that $|d(D)|=1$. Normal contribution of $D$ to the number of fixed points of $f^{n}$ would be 0 or 2 ; it has to be replaced by 1 . Thus there may be a correction $c_{n}$ from one or both poles, and $\left|c_{n}\right| \leq 2$. This proves (3.1). The inequality (3.2) follows immediately from (3.1).

Remark 3.2. It may happen that a pole does not belong to any band. Nevertheless, if it is a fixed point of $f^{n}$, we may wish to count its contribution to $c_{n}$ in order to improve the estimate.

Remark 3.3. Clearly, we have $k \geq k_{+}+k_{-}$. Also, $u \geq k_{+}+k_{-}$, so $u \geq\left|k_{+}-k_{-}\right|$. This makes the parts $k^{n}-\left(k_{+}+k_{-}\right)^{n}$ and $u^{n}-\left(k_{+}-k_{-}\right)^{n}$ of (3.1) (or (3.2)) in some sense independent of each other.

Corollary 3.4. Let $f \in \mathcal{L}$. With the notation of Theorem 3.1, if $k>k_{+}+k_{-}$, then

$$
\liminf _{n \rightarrow \infty} \frac{1}{n} \log N\left(f^{n}\right) \geq \log k(f)
$$

Similarly, if $u>\left|k_{+}-k_{-}\right|$, then

$$
\liminf _{n \rightarrow \infty} \frac{1}{n} \log N\left(f^{n}\right) \geq \log u(f) .
$$

Moreover, if $u=k_{-}-k_{+}$, then

$$
\limsup _{n \rightarrow \infty} \frac{1}{n} \log N\left(f^{n}\right) \geq \log u(f) .
$$

Let us discuss the possibilities occurring when we want to apply this corollary. First notice that $k>k_{+}+k_{-}$means that there is a band of latitude degree 0 . In this case (3.3) holds. The situation with $u$ is more complicated. If there is a band with the absolute value of the latitude degree larger than 1 , then $u>k_{+}+k_{-} \geq\left|k_{+}-k_{-}\right|$. If there is a band of latitude degree positive and a band of latitude degree negative, then $u \geq k_{+}+k_{-}>\left|k_{+}-k_{-}\right|$. Thus, in those two cases (3.4) holds. The remaining case is that either all bands have latitude degree 0 or 1 , or all bands have latitude degree 0 or -1 , and then (3.4) cannot be applied. However, if all bands have latitude degree 0 or -1 , then (3.5) holds.

The estimates from Theorem 3.1 and Corollary 3.4 are optimal, if we take into account only the information about the number of bands and their latitude degrees. For the relevant examples, see Section 5.

While the relation between the number of fixed points and the degree of the map will be investigated in the next section, we are already in a position to compare the growth rate of the number of fixed points of the iterates with the degree of the map. 
Theorem 3.5. If $f \in \mathcal{L}$ and $|\operatorname{deg}(f)|>1$, then

$$
\liminf _{n \rightarrow \infty} \frac{1}{n} \log N\left(f^{n}\right) \geq \log |\operatorname{deg}(f)| .
$$

Proof. If there is a band with the absolute value of the latitude degree larger than 1, or there is a band of latitude degree positive and a band of latitude degree negative, then, as we already noticed, (3.4) holds. This and the obvious inequality $u(f) \geq|\operatorname{deg}(f)|$ gives us (3.6). This leaves us with the case when either all bands have latitude degree 0 or 1 , or all bands have latitude degree 0 or -1 . If at least one band has latitude degree 0, then (3.3) holds, and since in this case $k(f)>u(f) \geq|\operatorname{deg}(f)|$, we also get (3.6). If all bands have latitude degree 1 or all bands have latitude degree -1 then, since $\Delta(B)$ alternates as we move $B$, we have $|\operatorname{deg}(f)| \leq 1$.

\section{Fixed POINTS AND DEGREE}

Now we forget about the iterates of $f \in \mathcal{L}$, and compare the degree of $f$ with the guaranteed number of its fixed points. The main result of this section is the following theorem. Observe that Theorem 3.5 is an immediate corollary to Theorem 4.1.

Theorem 4.1. If $f \in \mathcal{L}$ then

$$
N(f) \geq|\operatorname{deg}(f)+1| \text {. }
$$

We start with some preliminary considerations. Let us first make an assumption that in each band there is an invariant parallel, and count only fixed points in those invariant parallels. Denote the number of fixed points in a band $B$ by $N(B)$. Then by Lemma 2.4 we get

$$
N(B) \geq|d(B)-1|
$$

Consider a pair of bands, $A$ with $\Delta(A)=+1$ and $B$ with $\Delta(B)=-1$. If the number of bands is even, the set of bands can be partitioned into such pairs; if the number of bands is odd, there will be an additional single band. We will write $N(A, B)$ for $N(A)+N(B)$ and $\operatorname{deg}(A, B)$ for $\operatorname{deg}(A)+\operatorname{deg}(B)$.

Lemma 4.2. Let $A$ and $B$ be bands as above. Then

$$
N(A, B) \geq|\operatorname{deg}(A, B)| \text {. }
$$

Moreover, if $d(A) \leq 0$ and $d(B) \leq 0$ then

$$
N(A, B) \geq|\operatorname{deg}(A, B)|+2 .
$$

Proof. If both $d(A)$ and $d(B)$ are positive, then $N(A, B) \geq d(A)+d(B)-2$ and $\operatorname{deg}(A, B)=d(A)-d(B)$. If $d(A) \geq d(B)$ then

$$
N(A, B)-|\operatorname{deg}(A, B)| \geq d(A)+d(B)-2-(d(A)-d(B))=2 d(B)-2 \geq 0,
$$

so (4.3) holds. Similarly, if $d(B) \geq d(A)$, then (4.3) also holds.

If one of $d(A)$ and $d(B)$ is positive and the other one is nonpositive, then by (4.2)

$$
N(A, B) \geq|d(A)|+|d(B)|=|\operatorname{deg}(A, B)|,
$$

and (4.3) holds.

If both $d(A)$ and $d(B)$ are nonpositive, then $N(A, B) \geq|d(A)|+|d(B)|+2$ and $\operatorname{deg}(A, B)=|d(B)|-|d(A)|$. If $|d(A)| \geq|d(B)|$ then

$N(A, B)-|\operatorname{deg}(A, B)| \geq|d(A)|+|d(B)|+2-(|d(A)|-|d(B)|)=2|d(B)|+2 \geq 2$, 
so (4.4) holds. Similarly, if $|d(B)| \geq|d(A)|$, then (4.4) also holds.

Observe that Lemma 3 of [4] is incorrect (however, it is not essential in [4]). Namely, it may happen $f$ does not map a pole into a pole. The simplest example is a constant map, but using methods of Section 5 it is easy to construct latitudinal $C^{\infty}$ examples of any degrees. Assume that $X$ is a parallel that can be joined with this pole by an arc whose image under $f$ does not pass through a pole. Then the map $\left.f\right|_{X}$ as a map from a circle to a circle is homotopic to a constant map, so it has degree 0.

Lemma 4.3. Assume that the southmost band has positive sign, but the South Pole is not a fixed point. Then there is a fixed point between the South Pole and the leftmost band. The analogous statement is true for the northmost band and the North Pole.

Proof. Let $a \in(0,1]$ be the leftmost point such that $\varphi(a)=0$. The map $\varphi$ has a fixed point in $(0, a)$ (see Figure $2(\mathrm{~d})$ ), so there is an invariant parallel between the South Pole and the southmost band. As we observed, the degree of $f$ on this invariant parallel is 0 . Thus, there is a fixed point there.

Proof of Theorem 4.1. Assume first that there are no bands. By Lemma 2.2, $\operatorname{deg}(f)=$ 0 . If at least one of the poles is a fixed point, then (4.1) holds. If none of the poles is a fixed point, then we may assume that $1 \notin \varphi([0,1])$ and $\varphi(0) \neq 0$. The leftmost fixed point of $\varphi$ corresponds to an invariant parallel that can be joined to the South Pole with an arc whose image does not pass through a pole. Then the latitude degree on this parallel is 0 , so there is a fixed point in it. Hence, (4.1) holds.

Assume now that there is at least one band. We will consider various cases.

Case 1: the number of bands is even. By Lemma 4.2, if there is an invariant parallel in each band, then counting only fixed points in those invariant parallels, we get $N(f) \geq|\operatorname{deg}(f)|$. Moreover there is exactly one fixed pole. This gives us the estimate $N(f) \geq|\operatorname{deg}(f)|+1$, stronger than (4.1). However, we have to adjust this inequality if there is a band $B$ without an invariant parallel. This band has the fixed pole as one of the components of its boundary, and we have $\Delta(B)=1$ and $|d(B)|=1$. If $d(B)=1$, then we are done, because in our estimates in Lemma 4.2 $B$ did not contribute anything to the number of fixed points. Assume $d(B)=-1$. The contribution to the number of fixed points that we lose this way is 2 . If the next band $A$ (with $\Delta(A)=-1$ ) has latitude degree $d(A) \leq 0$, by Lemma 4.2 we get 2 extra fixed points, and we are done. Suppose that $d(A)>0$. Then $\operatorname{deg}(A, B) \leq-2$. If $\operatorname{deg}(f) \geq 0$, this means that considering $A$ and $B$ lowers the absolute value of the degree by at least 2 , and this compensates for the lost 2 fixed points. If $\operatorname{deg}(f)<0$, then $|\operatorname{deg}(f)+1|=|\operatorname{deg}(f)|-1$, so we need only the estimate $N(f) \geq|\operatorname{deg}(f)|-1$, which we have. This completes the proof in Case 1.

Case 2: the number of bands is odd. We again divide the set of bands into pairs, but we have additionally a single band $B$. If it has an invariant parallel, it contributes at least $|d(B)|-1$ fixed points if $d(B)>0$ and at least $|d(B)|+1$ fixed points if $d(B) \leq 0$. We have two subcases.

Subcase (a): no pole is fixed (the poles form a periodic orbit of period 2). Then every band has an invariant parallel, and we are done if $\operatorname{deg}(f)<0$. Assume $\operatorname{deg}(f) \geq$ 0 . Let $B$ the leftmost band. We have $\Delta(B)=-1$. If $d(B) \leq 0$, then $B$ contributes $|d(B)|+1$ fixed points, and we are done. Assume $d(B)>0$. Then $B$ contributes at 
least $|d(B)|-1$ to the number of fixed points and $-d(B)$ to the degree of $f$. The difference of those contributions is at least $2 d(B)-1 \geq 1$, and again we are done.

Subcase (b): both poles are fixed, so we get additional 2 fixed points, and (4.1) follows, except that one or two bands may have no invariant parallel. For such band $B$ we have $\Delta(B)=1$ and $|d(B)|=1$. If $d(B)=1$, we counted no fixed points contributed by $B$, so there are no problems with $B$. If $d(B)=-1$, we counted 2 fixed points. However, if this was the one band considered at the beginning of Case 2, we actually counted $|d(B)|-1=0$ fixed points, so again there are no problems with $B$. The only problem occurs when there are two such bands; we have to consider one of them as a member of a pair. However, then we argue as at the end of Case 1.

\section{EXAMPLES}

Although we are assuming only differentiability at the poles, we can construct relevant examples of class $C^{\infty}$. That is, for every finite sequences: $\Delta_{1}, \Delta_{2}, \ldots, \Delta_{n}$ of alternating numbers +1 and -1 , and $d_{1}, d_{2}, \ldots, d_{n}$ of integers, there exists a map $f \in \mathcal{L}$ of class $C^{\infty}$, whose bands are (from the South to North poles) $B_{1}, B_{2}, \ldots, B_{n}$ with $d\left(B_{i}\right)=d_{i}$ and $\Delta\left(B_{i}\right)=\Delta_{i}$. Of course we had to assume that plus and minus ones alternate, because of the shape of the graph of $\varphi$.

To construct such a map $f$ we first construct $\varphi$. We make it $C^{\infty}$ with the graph going up and down from level 0 to level 1 or vice versa (according to the sequence $\left(\Delta_{i}\right)$ ) and such that at the critical points derivatives of all orders are 0 . If $\varphi(0)=0$, we set in a local chart given by the stereographic projection from the North Pole $\varphi(t)=t^{d_{1}}$ for $t$ close to 0 , and we use an analogous formula if $\varphi(0)=1$. Also, we treat similarly the case $\varphi(1)=0$ or $\varphi(1)=1$. Moreover, we may assume that in each lap of $\varphi$ there is only one fixed point. An exception is when $n=1$ and $\Delta_{1}=1$. Then there are two fixed points, namely 0 and 1 on the unique lap of $\varphi$, and we drop the condition $\left|\varphi^{\prime}(1)\right|>1$. The construction of $\varphi$ satisfying the above conditions is standard.

Then we have to say how $f$ maps a parallel to a parallel. For this we work in a chart given by the stereographic projection from the North Pole to the complex plane $\mathbb{C}$. In this chart, parallels become circles centered at 0 . If a circle of radius $r$ belongs to a band of latitude degree $d$ and is mapped to a circle of radius $R$, this map is given by the formula $z \mapsto\left(R / r^{d}\right) z^{d}$ if $d \neq 1$, and $z \mapsto\left(R e^{i} / r\right) z$ if $d=1$ (observe that the multiplication by $e^{i}$ is an irrational rotation).

The only remaining thing is to show that the map $f$ constructed in such a way is of class $C^{\infty}$. This is true in the interior of each band by the construction. To get it at the components of the boundaries of the bands, we will show that there all partial derivatives of all orders are zero. Such a component is a parallel mapped to a pole. In suitable charts the map is $(x, y) \mapsto(\varphi(y) \cos (c x), \varphi(y) \sin (c x))$ for some constant $c$. The partial derivatives of all orders will be sums of terms such that each term contains $\varphi^{(k)}(y)$ for some $k \geq 0$. However, $\varphi^{(k)}(0)=0$ for all $k$, and this proves our claim.

This construction gives us the same number of fixed points for $f$ and its iterates as was used in the estimates in the preceding sections. In particular, this shows that the estimate (3.1) is sharp. The simple case with one band $B$, where $\Delta(B)=-1$, so $\operatorname{deg}(f)=-d(B)$, shows that the estimates from Theorem 4.1 and 3.5 are sharp. 


\section{REFERENCES}

[1] A. Katok, Lyapunov exponents, entropy, and periodic points for diffeomorphisms, Publ. Math. IHES, 51 (1980), 137-173.

[2] S. Kolyada and L. Snoha, Topological dynamics of triangular maps of the square, in: Iteration Theory (Batschuns, 1992), World Sci. Publ., River Edge, NJ, 1996, pp. 165-171.

[3] M. Misiurewicz and F. Przytycki, Topological entropy and degree of smooth mappings, Bull. Acad. Pol. Sci., Sér. sci. math., astr. et phys. 25 (1977), 573-574.

[4] C. Pugh and M. Shub, Periodic points on the 2-sphere, Discrete Cont. Dynam. Sys. 34 (2014), 1171-1182.

[5] M. Shub, Dynamical systems, filtration and entropy, Bull. Amer. Math. Soc. 80 (1974), $27-41$.

Department of Mathematical Sciences, IUPUi, 402 N. Blackford Street, IndiANAPOLIS, IN 46202

E-mail address: mmisiure@math.iupui.edu 